\title{
NORM ESTIMATES RELATING POLYNOMIALS AND THEIR DERIVATIVES
}

\author{
ZALMAN RUBINSTEIN
}

\begin{abstract}
Inequalities bounding $L_{p}(p>0)$ norms of a polynomial by suitable norms of the derivative of the polynomial and an auxiliary function are obtained for the classes of polynomials whose zeros lie on the unit circumference or in the interior or the exterior of the unit disk. All the inequalities are best possible and generalize several known estimates in particular cases.
\end{abstract}

1. Introduction. We shall call a polynomial $P(z)=\sum_{k=0}^{n} a_{k} z^{k}=a_{n} \Pi_{k=1}^{n}\left(z-z_{k}\right)$ of degree $n$ a $C$-polynomial if all its zeros lie on the unit circumference $C=\{z|| z \mid$ $=1\} . D$ and $D^{*}$ shall denote the closed interior and the closed exterior of $C$. $D$-polynomials and $D^{*}$-polynomials are defined analogously to $C$-polynomials. For any polynomial $P(z)$ of degree $n$ we set $P^{*}(z)=z^{n} \overline{P(1 / \bar{z})}$.

A polynomial $P(z)$ is called self-inversive if $\left\{z_{1}, z_{2}, \ldots, z_{n}\right\}=$ $\left\{1 / \bar{z}_{1}, 1 / \bar{z}_{2}, \ldots, 1 / \bar{z}_{n}\right\}$. It is well known ([5, Lemma 2], [7, equation (6)]) that for self-inversive polynomials the identity

$$
P^{\prime *}(z)=n P(z) / u(1+w(z))
$$

holds for $|z|=1$, where $|u|=1,|w(z)|=1$ and $w(0)=0$. If $P(z)$ is a $C$-polynomial the function $w(z)$ is analytic in the unit disk and thus satisfies the conditions of Schwarz' lemma.

We shall make use of the following integrals

$$
C q=\int_{0}^{2 \pi}\left|1+e^{i \theta}\right|^{q} d \theta=\frac{2^{q+1} \sqrt{\pi} \Gamma\left(\frac{1}{2} q+\frac{1}{2}\right)}{\Gamma\left(\frac{1}{2} q+1\right)} \text { for } q>-1 .
$$

From the well-known integral representation of the Legendre polynomials

$$
P_{n}(z)=\frac{1}{\pi} \int_{0}^{\pi}\left(z+\sqrt{z^{2}-1} \cos \theta\right)^{n} d \theta
$$

for $\operatorname{Re} z>0, \arg \left(z+\sqrt{z^{2}-1} \cos \theta\right)=\arg z$ for $\theta=\pi / 2$ [2, p. 1017] one obtains the formula

$$
\int_{0}^{2 \pi}\left(1+r^{2}+2 r \cos \theta\right)^{n} d \theta=2 \pi\left|1-r^{2}\right|^{n} P_{n}\left(\frac{1+r^{2}}{\left|1-r^{2}\right|}\right)
$$

for $r>0$. For an integrable function $f$ defined on the unit circumference we shall use the notation $\|f\|_{p}(p>0)$ for the integral $\|f\|_{p}=\left(\int_{0}^{2 \pi}\left|f\left(e^{i \theta}\right)\right|^{p} d \theta\right)^{1 / p}$. However

Received by the editors June 13, 1979.

1980 Mathematics Subject Classification. Primary 41A44; Secondary 41A17.

$K e y$ words and phrases. $L_{p}$-norm, inequality, $C$-polynomial.

(c) 1980 American Mathematical Society $0002-9939 / 80 / 0000-0413 / \$ 02.25$ 
all the main results apply to the more customary norm $\left((1 / 2 \pi) \int_{0}^{2 \pi}\left|f\left(e^{i \theta}\right)\right|^{p} d \theta\right)^{1 / p}$ as well.

The purpose of this note is to prove several inequalities relating the various norms of a polynomial and its derivative. The classes treated here are the $C, D$, and $D^{*}$ polynomials. All the inequalities are sharp with the exact meaning indicated in each case.

Inequalities of this type have been extensively studied. The departure of this note is in two respects.

In most well-know inequalities the norm of the derivative or some function of it is dominated by a certain norm of the polynomial. The results presented here are in the reverse direction, namely certain norms of the polynomial are dominated by suitable norms of the derivative.

Secondly these inequalities contain in particular $L_{p}$ norms where $p<1$. Inequalities of this type have been studied only recently in [4] and [6] with the aim of extending the well-known Bernstein's inequality for trigonometric polynomials to $L_{p}, 0<p<1$. This conjecture can be formulated as $\left\|T_{n}^{\prime}(\theta)\right\|_{p}<n\left\|T_{n}(\theta)\right\|_{p}$ for all trigonometric polynomials of degree $n$.

\section{The main results.}

THEOREM 1. Let $p$ and $q$ satisfy $1 / p+1 / q=1, p>1, q>1$ and let $r>0$. Then for every $C$-polynomial $P(z)$ of degree $n$ we have

$$
n\|P\|_{r} \leqslant\left\|P^{\prime}\right\|_{r p}\|1+z\|_{r q} .
$$

Equality is attained for all $r$ by $P(z)=(z+1)^{n}$ and $q=n$.

Proof. Since $\left|P^{\prime *}\left(e^{i \theta}\right)\right|=\left|P^{\prime}\left(e^{i \theta}\right)\right|$ for real $\theta$ we have by Hölder's inequality and (1)

$$
\begin{aligned}
\int_{0}^{2 \pi}\left|P\left(e^{i \theta}\right)\right|^{r} d \theta & \leqslant\left(\int_{0}^{2 \pi} \frac{\left|P\left(e^{i \theta}\right)\right|^{r p}}{\left|1+w\left(e^{i \theta}\right)\right|^{r p}} d \theta\right)^{1 / p}\left(\int_{0}^{2 \pi}\left|1+w\left(e^{i \theta}\right)\right|^{r q} d \theta\right)^{1 / q} \\
& \leqslant C_{r q}^{1 / q_{n}-r}\left(\int_{0}^{2 \pi}\left|P^{\prime}\left(e^{i \theta}\right)\right|^{r p} d \theta\right)^{1 / p}
\end{aligned}
$$

The last inequality follows by a theorem of Rogosinski [3, Theorem 18.5.3]. Thus

$$
n^{r} \int_{0}^{2 \pi}\left|P\left(e^{i \theta}\right)\right|^{r} d \theta \leqslant\left(\int_{0}^{2 \pi}\left|P^{\prime}\left(e^{i \theta}\right)\right|^{r p} d \theta\right)^{1 / p} C_{r q}^{1 / q}
$$

Taking the $r$ th root on both sides of the last equation we arrive at (4). If $P(z)=(z+1)^{n},\|P\|_{r}=C_{n r}^{1 / r},\left\|P^{\prime}\right\|_{r p}=n C_{(n-1) r p}^{1 / r p}$ and $\|1+z\|_{r q}=C_{r q}^{1 / r q}$. Therefore (4) reduces to $C_{n r}^{1 / r} \leqslant C_{(n-1) r p}^{1 / r p} C_{r q}^{1 / r q}$. One verifies that the last inequality becomes an equality for $q=n, p=n /(n-1)$ and arbitrary $r$ since $1 / p+$ $1 / q=1$.

Particular cases of Theorem 1 are also of interest. We examine a few.

(a) $p \rightarrow 1, q \rightarrow \infty$.

$$
n\|P\|_{r} \leqslant\left\|P^{\prime}\right\|_{r}\|1+z\|_{\infty}=2\left\|P^{\prime}\right\|_{r} .
$$


On the other hand an inequality of de Bruijn [1] for polynomials $P(z) \neq 0$ in

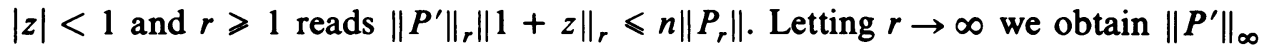
$=(n / 2)\|P\|_{\infty}$. This equality was established for self-inversive polynomials by O'Hara and Rodriguez [5]. The corresponding inequality was conjectured by $\mathbf{P}$. Erdös and proved by P. D. Lax (see [1], [7]).

(b) $q \rightarrow 1, p \rightarrow \infty$.

$$
n\|P\|_{r} \leqslant\left\|P^{\prime}\right\|_{\infty}\|1+z\|_{r} .
$$

This inequality becomes an equality for $P(z)=z^{n}+1$ since $\left\|1+z^{n}\right\|_{r}=$ $\|1+z\|_{r}$.

(c) $p=q=2$.

$$
n\|P\|_{r} \leqslant\left\|P^{\prime}\right\|_{2 r}\|1+z\|_{2 r}
$$

Finally

(d) $r=q=1, p \rightarrow \infty$. By (a) we have

$$
\|P\|_{1} \leqslant(8 / n)\left\|P^{\prime}\right\|_{\infty}=4\|P\|_{\infty} \text {. }
$$

The last inequality was applied by Saff and Sheil-Small to solve a conjecture of Erdös [7] regarding trigonometric polynomials.

In the proof of the next theorem we shall need the following.

LEMma. Let $f(t)$ and $g(t)$ be continuous functions defined on the unit circumference. Let $\delta$ be the closure of the subset of $[0,1]$ defined by

$$
\left\{x=\frac{|f(t)|^{2}-|g(t)|^{2}}{|f(t)|^{2}+|g(t)|^{2}},|t|=1,|f(t)|^{2}+|g(t)|^{2}>0\right\} .
$$

Furthermore, let $M_{m}=\operatorname{Max}_{x \in \delta} P_{m}^{*}(x), \mu_{m}=\operatorname{Min}_{x \in \delta} P_{m}^{*}(x)$, where $P_{m}(x)$ is the Legendre polynomial of degree $m$. Then

$$
\begin{aligned}
\mu_{m}\left(\left|f\left(e^{i \theta}\right)\right|^{2}+\left|g\left(e^{i \theta}\right)\right|^{2}\right)^{m} & \leqslant \frac{1}{2 \pi} \int_{0}^{2 \pi}\left|f\left(e^{i \theta}\right)+e^{i \alpha} g\left(e^{i \theta}\right)\right|^{2 m} d \alpha \\
& \leqslant M_{m}\left(\left|f\left(e^{i \theta}\right)\right|^{2}+\left.g\left(e^{i \theta}\right)\right|^{2}\right)^{m}
\end{aligned}
$$

Proof. Set $r=\left|g\left(e^{i \theta}\right)\right| /\left|f\left(e^{i \theta}\right)\right|, f\left(e^{i \theta}\right) \neq 0$. By (3)

$$
\begin{aligned}
\int_{0}^{2 \pi}\left|f\left(e^{i \theta}\right)+e^{i \alpha} g\left(e^{i \theta}\right)\right|^{2 m} d \alpha=\left|f\left(e^{i \theta}\right)\right|^{2 m} \int_{0}^{2 \pi}\left(1+r^{2}+2 r \cos \alpha\right)^{m} d \alpha \\
=2 \pi\left|f\left(e^{i \theta}\right)\right|^{2 m}\left|1-r^{2}\right|^{m} P_{m}\left(\frac{1+r^{2}}{\left|1-r^{2}\right|}\right) \\
=2 \pi\left|f\left(e^{i \theta}\right)\right|^{2 m}\left(1+r^{2}\right)^{m} P_{m}^{*}\left(\frac{\left|1-r^{2}\right|}{1+r^{2}}\right) \\
=2 \pi\left(\left|f\left(e^{i \theta}\right)\right|^{2}+\left|g\left(e^{i \theta}\right)\right|^{2}\right)^{m} P_{m}^{*}\left(\frac{\left.|| f\left(e^{i \theta}\right)\right|^{2}-\left|g\left(e^{i \theta}\right)\right|^{2} \mid}{\left|f\left(e^{i \theta}\right)\right|^{2}+\left|g\left(e^{i \theta}\right)\right|^{2}}\right) .
\end{aligned}
$$

This implies (5). If $g\left(e^{i \theta}\right) \neq 0$ we define $r=f\left(e^{i \theta}\right) / g\left(e^{i \theta}\right)$ and proceed as before. Obviously we can disregard points $\theta$ for which $f\left(e^{i \theta}\right)=g\left(e^{i \theta}\right)=0$. We remark that 
if $\left|f\left(e^{i \theta}\right)\right|=\left|g\left(e^{i \theta}\right)\right|$ for $0 \leqslant \theta \leqslant 2 \pi$, then $\delta=\{0\}$ and $M_{m}=\mu_{m}=(2 m) ! /\left(m !^{2} 2^{m}\right)$ $=P_{m}^{*}(0)=C_{2 m} /(2 \pi) 2^{-m}$. In general it follows from the integral representation of $P_{m}(z)$ that $M_{m} \leqslant 2^{m}$. The case $\delta=\{0\}$ occurs when $f=P$ and $g=P^{*}$ for an arbitrary polynomial $P$ or if $f=P^{\prime}$ and $g=P^{* \prime}$ and $P$ is self-inversive, since for such a polynomial $P^{*}(z)=e^{i \alpha} P(z)$. We may also note that inequality (5) establishes the equivalence between the norms $\left\|f\left(e^{i \theta}\right)+e^{i \alpha} g\left(e^{i \theta}\right)\right\|_{2 m}(\theta)$ and the Euclidean norm $\left|f\left(e^{i \theta}\right)+i g\left(e^{i \theta}\right)\right|_{2}(\theta)$. Now we generalize some of the previous results to a wider class of polynomials, namely for those which do not vanish either in the interior or the exterior of the unit disk.

THEOREM 2. Let $R(z)$ be a polynomial of degree $n$ whose zeros all lie in $D$ or $D^{*}$; then

$$
\|R\|_{\infty} \leqslant \frac{\sqrt{2}}{n}\left\|\left(\left|R^{\prime}\left(e^{i \theta}\right)\right|^{2}+\left|R^{* \prime}\left(e^{i \theta}\right)\right|^{2}\right)^{1 / 2}\right\|_{\infty} .
$$

The number $\sqrt{2} / n$ cannot be replaced by any other smaller number.

Proof. Since $R(z)$ is a $D$ or $D^{*}$-polynomial it is easy to see that $P(z)$ defined by $P(z)=R(z)+e^{i \alpha} R^{*}(z)$ is a $C$-polynomial for all real $\alpha$. Thus we may apply inequality (4) to $P(z)$ in the form

$$
n^{2 m / p}\|P\|_{2 m / p}^{2 m / p} \leqslant\left\|P^{\prime}\right\|_{2 m / p}^{2 m / p}\|1+z\|_{2 m q / p}^{2 m / p}
$$

where $p$ and $q$ exceed 1 and $m$ is a positive integer. We now evaluate the integrals on both sides of (7) separately after integrating with respect to $\alpha$ in the interval $[0,2 \pi]$.

The first integral in (7) can be evaluated as follows:

$$
\begin{aligned}
\int_{0}^{2 \pi} \int_{0}^{2 \pi}\left|P\left(e^{i \theta}\right)\right|^{2 m / p} d \theta d \alpha & =\int_{0}^{2 \pi} d \theta \int_{0}^{2 \pi}\left|R\left(e^{i \theta}\right)+e^{i \alpha} R^{*}\left(e^{i \theta}\right)\right|^{2 m / p} d \alpha \\
& =C_{2 m / p} \int_{0}^{2 \pi}\left|R\left(e^{i \theta}\right)\right|^{2 m / p} d \theta .
\end{aligned}
$$

The second integral in (7) can be estimated by Hölder's inequality and the lemma

$$
\begin{gathered}
\int_{0}^{2 \pi}\left(\int_{0}^{2 \pi}\left|P^{\prime}\left(e^{i \theta}\right)\right|^{2 m} d \theta\right)^{1 / p} d \alpha \\
\leqslant(2 \pi)^{1 / q}\left(\int_{0}^{2 \pi} d \theta \int_{0}^{2 \pi}\left|R^{\prime}\left(e^{i \theta}\right)+e^{i \alpha} R^{* \prime}\left(e^{i \theta}\right)\right|^{2 m} d \alpha\right)^{1 / p} \\
\leqslant 2 \pi M_{m}^{1 / p}\left(\int_{0}^{2 \pi}\left(\left|R^{\prime}\left(e^{i \theta}\right)\right|^{2}+\left|R^{*^{\prime}}\left(e^{i \theta}\right)\right|^{2}\right)^{m} d \theta\right)^{1 / p}
\end{gathered}
$$

Substituting into (7) and raising to power $p / 2 m$ we have

$$
\begin{aligned}
\|R\|_{2 m / p} \leqslant & \frac{1}{n}(2 \pi)^{p / 2 m} C_{2 m}^{-p / p} / 2 m M_{m}^{1 / 2 m} \\
& \cdot\left(\int_{0}^{2 \pi}\left(\left|R^{\prime}\left(e^{i \theta}\right)\right|^{2}+\left|R^{*^{\prime}}\left(e^{i \theta}\right)\right|^{2}\right)^{m} d \theta\right)^{1 / 2 m}\|1+z\|_{2 m q / p} .
\end{aligned}
$$

Finally let $m \rightarrow \infty$. As was remarked following the proof of the lemma $M_{m}<2^{m}$. Also $C_{2 m}^{-p / p} \rightarrow 1 / 2$ and $\|1+z\|_{2 m q / p} \rightarrow 2$. This implies (6). 
If $R(z)$ is a $C$-polynomial then (6) reduces to the inequality $\|R\|_{\infty}<$ $(2 / n)\left\|R^{\prime}\right\|_{\infty}$ which becomes an equality in view of the considerations in example (a) following the proof of Theorem 1 . This shows that the constant $\sqrt{2} / n$ cannot be replaced by a smaller number. We conclude with two remarks.

(a) It may be worthwhile to mention that we do not have unique extremal polynomials. For example the equality $\|R\|_{\infty}=(2 / n)\left\|R^{\prime}\right\|_{\infty}$ is satisfied by all polynomials of the form $\left(z^{k}-1\right)^{n / k}$ where $k$ is any positive integer dividing $n$.

(b) The function $R_{1}\left(e^{i \theta}\right)=\operatorname{Max}\left(\left|R^{\prime}\left(e^{i \theta}\right)\right|,\left|R^{* \prime}\left(e^{i \theta}\right)\right|\right)$ plays, in view of the preceding results, the role of the modulus of the derivative for $D$ - or $D^{*}$-polynomials and reduces to it in the particular case of $C$-polynomials.

\section{REFERENCES}

1. N. G. de Bruijn, Inequalities concerning polynomials in the complex domain, Nederl. Akad. Wetensch. Proc. Ser. A 50 (1947), 1265-1272 = Indag. Math. 9 (1947), 591-598.

2. I. S. Gradshteyn and I. M. Ryzhik, Table of integrals, series and products, Academic Press, New York, 1965.

3. E. Hille, Analytic function theory. II, Ginn, Boston, New York and Toronto, 1962.

4. A. Máté and P. Nevai, Bernstein's inequality in $L^{p}$ for $0<p<1$ and $(C, 1)$ bounds for orthogonal polynomials, Notices Amer. Math. Soc. 26 (1979), A-210.

5. P. J. O'Hara, and R. S. Rodriguez, Some properties of self-inversive polynomials, Proc. Amer. Math. Soc. 44 (1974), 331-335.

6. P. Oswald, Some inequalities for trigonometric polynomials in $L^{p}, 0<p<1$, Izv. Vuzov $=$ Izv. Vysš. Učebn. Zaved. Matematica 20 (1976), 65-75.

7. E. B. Saff and T. Sheil-Small, Coefficient and integral mean estimates for algebraic and trigonometric polynomials with restricted zeros, J. London Math. Soc. 9 (1974), 16-22.

Department of Mathematics, University of Michigan, Ann Arbor, Michigan 48109

DePARTMENT OF MATHEMATICS, University OF HAIFA, HAIFA, ISRAEL (Current address) 